\title{
Dihydroartemisinin attenuates osteoarthritis by inhibiting abnormal bone remodeling and angiogenesis in subchondral bone
}

\author{
LONG MA $^{1,2}$, XIN ZHAO $^{1,2}$, YIBIN LIU $^{1}$, JIANG WU $^{2}$, XIAOCHUN YANG $^{1,2}$ and QUNHUA JIN $^{2}$ \\ ${ }^{1}$ Ningxia Medical University, ${ }^{2}$ Orthopedics Ward 3, The General Hospital of Ningxia Medical University, \\ Yinchuan, Ningxia Hui Autonomous Region 750004, P.R. China
}

Received April 23, 2020; Accepted December 15, 2020

DOI: $10.3892 /$ ijmm.2021.4855

\begin{abstract}
The present study aimed to investigate whether dihydroartemisinin (DHA) alleviates osteoarthritis (OA) in a mouse model of OA. Ten-week-old female C57BL/6j mice were used to establish OA models by anterior cruciate ligament transection (ACLT) and ovariectomized (OVX). DHA was then used to treat the OA in the ACLT and OVX mice. Safranin O-fast green staining and Osteoarthritis Research Society International (OARSI)-modified Mankin scores were used to grade articular cartilage degeneration. Expression of metalloproteinase-13 (MMP-13) and vascular endothelial growth factor (VEGF) in the articular cartilage and leukemia inhibitory factor (LIF), sclerostin, and $\beta$-catenin in the subchondral bone were analyzed by immunohistochemistry. Expression of RANKL and CD31 were detected by immunofluorescence. Micro-computed tomography was used to ascertain alterations in the microarchitecture of the subchondral bone. The results demonstrated that DHA decreased MMP-13 and VEGF expression in the articular cartilage. DHA decreased OARSI scores and reduced articular cartilage degeneration. In addition, DHA reduced abnormal subchondral bone remodeling, as demonstrated by a reduction in trabecular separation (Tb.Sp), increased bone volume fractions (BV/TV), as well as bone mineral densities (BMD) compared with the ACLT+vehicle group and the OVX+vehicle group. Furthermore, DHA decreased the inhibition of sclerostin through reduction of LIF secretion by osteoclasts and, hence, attenuated aberrant bone remodeling and inhibited angiogenesis in subchondral bone, further reducing the progression of OA. The present study demonstrated that DHA attenuated OA by inhibiting abnormal bone remodeling and angiogenesis in subchondral bone, which may be a potential therapeutic target for this disease.
\end{abstract}

Correspondence to: Professor Qunhua Jin, Orthopedics Ward 3, The General Hospital of Ningxia Medical University, 804 Shengli South Street, Yinchuan, Ningxia Hui Autonomous Region 750004, P.R. China

E-mail: jinqunhua163@163.com

Key words: osteoarthritis, subchondral bone, articular cartilage, angiogenesis, dihydroartemisinin, leukemia inhibitory factor

\section{Introduction}

Osteoarthritis is the most common degenerative joint disease, causing pain and dysfunction in numerous joints (1). Due to its complex pathogenesis, there is currently no effective treatment for osteoarthritis (OA) (1). Therefore, a better understanding of the pathogenesis of OA would be of great significance to aid in its early prevention and treatment.

The subchondral bone is located below the articular cartilage, which plays a significant role in disease pathogenesis (2). Changes in the microstructure of the subchondral bone can be observed earlier than damage to the articular cartilage $(3,4)$. Under physiological conditions, bone remodeling maintains bone tissue integrity through the coupling of osteoclast-mediated resorption and osteoblast-mediated formation of bone (5). Abnormal bone remodeling causes microstructural destruction of the subchondral bone, altering its mechanical properties (6). Additionally, articular cartilage does not withstand shear stress during joint movement, degenerating as a result $(2,7)$. Increased subchondral bone remodeling is characterized by increased bone loss, decreased bone density, and deterioration of its microstructure, which leads to articular cartilage degeneration (8). Bone resorption due to osteoclasts has been demonstrated to significantly increase in anterior cruciate ligament transection (ACLT) and ovariectomized (OVX) models of OA. A reduction in articular cartilage degeneration can be observed when osteoclast-mediated bone resorption is inhibited using alendronate $(9,10)$. In OVX mice, estrogen deficiency causes osteoclast-mediated bone resorption, increased subchondral bone remodeling, and deterioration of the subchondral bone microstructure, resulting in degeneration of the articular cartilage, which establishes an osteoporosis-associated OA mouse model induced by ovariectomy $(9,11,12)$. These studies suggest that abnormal subchondral bone remodeling destroys subchondral bone microstructure, inducing degeneration of the articular cartilage.

Sclerostin, a potent inhibitor of the Wnt/ $\beta$-catenin pathway, is secreted by osteocytes and inhibits osteoblast-mediated bone formation $(13,14)$. As OA develops, concentrations of sclerostin in the subchondral bone gradually decrease, activating the Wnt/ $\beta$-catenian pathway that promotes bone formation and accelerates cartilage degradation (15). Previous studies have suggested that mechanical stress leads to a decrease in sclerostin in subchondral bone, however, the mechanism by 
which sclerostin levels decline in the subchondral bone remains entirely unclear (16-18). LIF is secreted by osteoclasts and acts between bone resorption and formation as a regulator of bone remodelling (19). Studies on osteoporosis have demonstrated that osteoclasts oversecrete LIF, inhibiting the expression of sclerostin and, thus, promoting abnormal bone formation and mediating abnormal bone remodelling (20). Inhibition of osteoclastogenesis can reduce the secretion of LIF, thereby reducing abnormal bone remodeling in osteoporosis (20). However, the role of LIF in the abnormal remodeling of the subchondral bone during OA remains unclear.

Abnormal angiogenesis of the subchondral bone is an important pathological feature that leads to abnormal bone remodeling in OA (21). In a mouse model of OA established by ACLT and OVX, the vascular endothelial progenitor cell marker CD31 was significantly increased in the subchondral bone $(12,22-24)$ and the expression of the catabolic factors, MMP-13 and VEGF were also significantly increased, resulting in the articular cartilage being severely damaged. Inhibition of the abnormal angiogenesis of the subchondral bone alleviates articular cartilage degeneration (22-24).

Artemisinin, a well-known antimalarial drug, is a sesquiterpene lactone isolated from Artemisia (25). Dihydroartemisinin (DHA) is a semisynthetic derivative of artemisinin that has fewer side effects (26). DHA reduces bone loss by inhibiting receptor activator of nuclear factor $\kappa \mathrm{B}$ ligand (RANKL)-induced osteoclastogenesis in OVX mice and in a wear particle-induced mouse osteolysis model $(27,28)$. In addition, DHA has been revealed to have an antiangiogenic effect $(27,28)$. However, the role of DHA in the abnormal bone remodeling and angiogenesis of OA remains unclear. The aim of this study was to investigate the potential role of DHA in alleviating abnormal subchondral bone remodeling and angiogenesis in order to reduce articular cartilage degeneration in a mouse model of OA.

\section{Materials and methods}

Ethics statement. All procedures and protocols were approved by the Scientific Research Ethics Committee of the General Hospital of Ningxia Medical University (protocol no. 2016-147). All experiments were performed in accordance with the principles and guidelines of the National Institutes of Health Guide for Care and Use of Laboratory Animals.

Animals. A total of 120 female C57BL/6j mice (weight, 19-20 g), ten weeks old, were used in the present study. All mice were housed at a constant temperature $\left(25^{\circ} \mathrm{C}\right)$, at $55 \%$ humidity on a 12-h light/dark cycle with free access to food and water. The mice were anesthetized with pentobarbital sodium salt $(60 \mathrm{mg} / \mathrm{kg})$ by intraperitoneal injection. The schematic of the experimental protocol is listed in Fig. 1.

Sixty mice were randomly divided into 3 groups: A sham-operated group $(n=20)$, ACLT+vehicle group $(n=20)$, and ACLT+DHA group $(n=20)$. Surgery was performed on the right knees of each mouse as previously described (29). In the two ACLT groups, the right knee joint capsule was exposed using a medial parapatellar approach and then the anterior cruciate ligament was transected with micro-scissors, prior to closing the joint capsule and skin. The same procedure was performed in the sham-operated group with the exception of the transection of the ACL. The ACLT+DHA group was treated with DHA (product no. D7439; Sigma-Aldrich; Merck KGaA) by intraperitoneal injection ( $1 \mathrm{mg} / \mathrm{kg} / 2$ days) from the first postoperative day until sacrifice (Fig. 1A). Flow displacement rate of $\mathrm{CO}_{2}$ used for euthanasia was $30 \%$. Mouse euthanasia was confirmed, including lack of pulse, breathing, corneal reflex, and response to firm toe pinch. DHA was prepared according to the literature (30), and the method was as follows: $25 \mathrm{mg}$ of DHA was weighed in a precision electronic balance, and dissolved in $1 \mathrm{ml}$ of prepared DMSO, and then diluted in $99 \mathrm{ml}$ sterile PBS solution to prepare $1 \mathrm{mg} / \mathrm{kg}$ DHA. The sham and vehicle treatment groups were administered solvent as a vehicle, using the same dose, frequency and duration as the DHA group.

Sixty mice were randomly divided into 3 groups: A sham-operated group $(n=20)$, OVX+vehicle group $(n=20)$, and OVX+DHA group $(n=20)$. According to the literature, the mice were operated from the abdominal cavity through the back to remove the bilateral ovaries $(12,31)$. The same procedure was performed in the sham-operated group, except that the bilateral ovaries were only removed. The OVX+DHA group was treated with DHA from the first day after operation (1 mg/kg/2 days) until sacrifice (Fig. 1B). Ovietectomy was performed under anaesthesia. The mice were anesthetized with pentobarbital sodium salt $(60 \mathrm{mg} / \mathrm{kg})$ by intraperitoneal injection. The method of euthanasia and the configuration of DHA were the same as those of the ACLT mice. The sham operation and carrier groups used the same dose, frequency, and duration as the DHA group. Ten mice were separately euthanized at 4 or 8 weeks after surgery in each group by carbon dioxide inhalation, followed immediate harvesting of the right knees for subsequent analysis.

Histology. Harvested knees were fixed in $4 \%$ paraformaldehyde for $24 \mathrm{~h}$ at $4^{\circ} \mathrm{C}$ then decalcified in $10 \%$ EDTA (pH 7.4) for 3 weeks prior to embedding in paraffin. The medial compartment of the knee joints were cut into $4-\mu$ m-thick sections along the sagittal plane and stained with safranin O-fast green at $25^{\circ} \mathrm{C}$ in the laboratory. Deparaffinization of the slides was performed in xylene two times for $10 \mathrm{~min}$, followed by hydration in $100 \%$ alcohol twice for $5 \mathrm{~min}, 95 \%$ alcohol for $2 \mathrm{~min}$ and $80 \%$ alcohol for $2 \mathrm{~min}$. Hematoxylin was added to slides for $3 \mathrm{~min}$ prior to hydrating the slides gently in running water for $10 \mathrm{~min}$. Slides were then stained with $0.2 \%$ Fast Green (product no. F7252; Sigma-Aldrich; Merck KGaA) for $3 \mathrm{~min}$, and then subjected to $1 \%$ acetic acid for $5 \mathrm{sec}, 0.1 \%$ Safranin O (product no. S8884; Sigma-Aldrich; Merck KGaA) for $3 \mathrm{~min}$. Slides were hydrated in $95 \%$ alcohol for $5 \mathrm{sec}, 100 \%$ alcohol twice for $15 \mathrm{sec}$, followed by 2 changes in xylene prior to cover-slipping the slides. Regions from three slides per mouse were imaged by light microscopy at a magnification of x100 and three fields per region were randomly selected per slide. The Osteoarthritis Research Society International (OARSI)-modified Mankin score was used to perform a histopathological grade assessment of the cartilage.

Immunohistochemistry and immunofluorescence. Standard immunostaining was conducted in the present study. Sagittal 
A

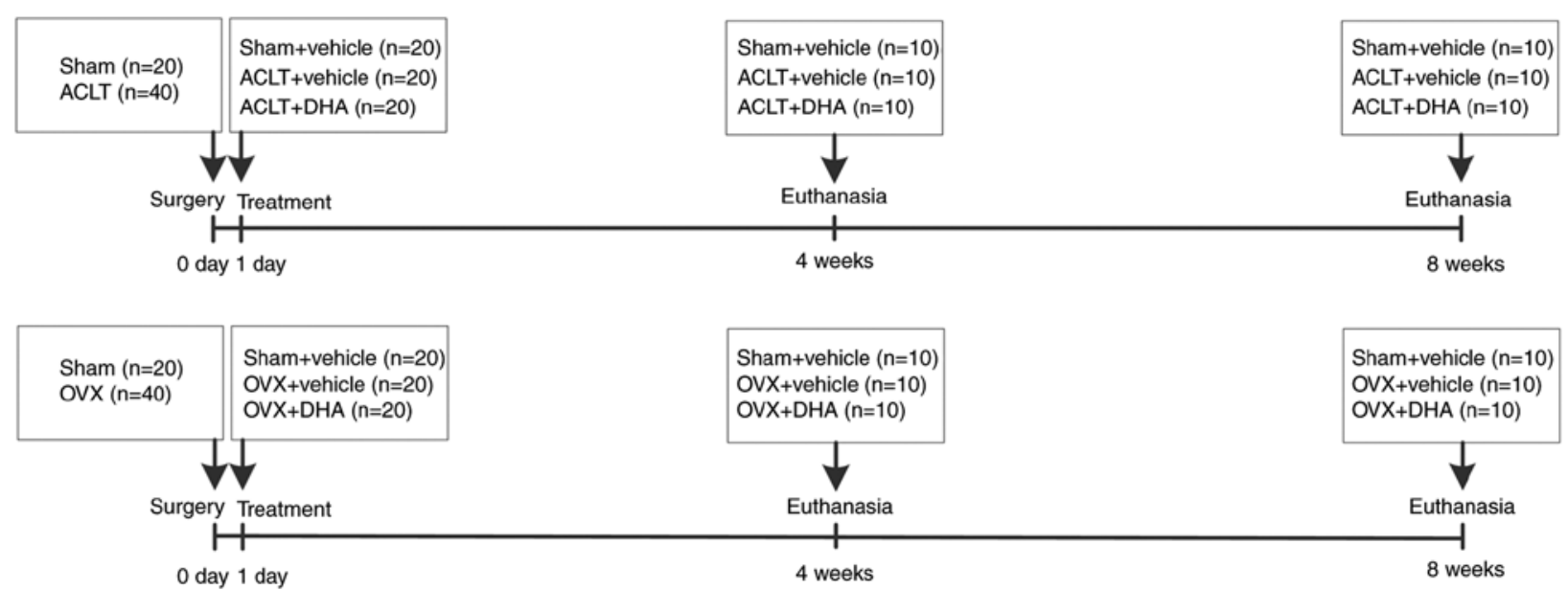

Figure 1. Schematic of the experimental protocol. (A) Schematic of the experimental protocol of ACLT mice. (B) Schematic of the experimental protocol of OVX mice. ACLT, anterior cruciate ligament transection; OVX, ovariectomized.

sections that had been paraffin-embedded were incubated overnight at $4^{\circ} \mathrm{C}$ with primary antibodies against matrix metalloproteinase-13 (MMP-13) (1:100; product code ab39012; Abcam), vascular endothelial growth factor A (VEGFA) (1:100; product code ab52917; Abcam), leukemia inhibitory factor (LIF) (1:100; cat. no. AB-449-NA; R\&D Systems, Inc.), sclerostin (1:50; product code ab63097; Abcam), $\beta$-catenin (1:100; cat. no. 51067-2-AP; ProteinTech Group, Inc.), RANKL (1:100; product code ab216484; Abcam) and CD31 (1:25; product code ab28364; Abcam). For immunohistochemical staining, sections were processed using a two-step IHC detection reagent (ZSGB-Bio; OriGene Technologies, Inc.). Briefly, sections were incubated with reaction enhancement solution (reagent 1) for $20 \mathrm{~min}$ at $37^{\circ} \mathrm{C}$ and then with enhanced enzyme-labeled goat anti-rabbit IgG polymer (reagent 2 ) for $40 \mathrm{~min}$ at $37^{\circ} \mathrm{C}$. The sections were then developed using 3,3'-diaminobenzidine (DAB) (ZSGB-Bio; OriGene Technologies, Inc.), followed by counterstaining with hematoxylin (ZSGB-Bio; OriGene Technologies, Inc.). For immunofluorescence staining, sections were incubated with Alexa Fluor ${ }^{\circledR} 488$ goat anti-rabbit secondary antibody (1:500; product code ab150077; Abcam) for $1 \mathrm{~h}$ at $37^{\circ} \mathrm{C}$ in the dark. Regions from three slides per mouse were imaged by fluorescence microscopy at a magnification of $x 400$ and three fields per region were randomly selected per slide. Image-Pro Plus 6.0 (Media Cybernetics, Inc.) was used to count chondrocytes within the entire tibial articular cartilage and all cells within the entire tibial subchondral bone which exhibited positive staining for each antibody.

Micro-computed tomography (CT) analysis. Harvested knee joints were dissected free of soft tissue, fixed overnight in $70 \%$ ethanol at $4^{\circ} \mathrm{C}$ and analyzed by micro-CT (SkyScan 1176; Bruker micro-CT) at a resolution of $9 \mu \mathrm{m} /$ pixel. The micro-CT scans were acquired over an exposure time of $900 \mathrm{~ms}$, a voltage of $50 \mathrm{kV}$ and a current of $500 \mu \mathrm{A}$. Images were reconstructed by NRecon version 1.1.11 (Bruker micro-CT) and analyzed by CTAn, v1.15 (SkyScan1176 in vivo micro-CT; Bruker). A sagittal view of the entire medial compartment of the tibial subchondral bone was used for 3D histomorphometric analysis, with bone volume/tissue volume (BV/TV, \%), trabecular separation (Tb.Sp), and BMD extracted for performing comparisons.

Statistical analysis. Data were analyzed using one-way and two-way factorial design analyses of the variations followed by Student-Newman-Keuls and Bonferroni post hoc tests. Data are presented as the means \pm SD. For OARSI scores, data were analyzed using Kruskal-Wallis test followed by Dunn's test. $\mathrm{P}<0.05$ was considered to indicate a statistically significant difference. GraphPad Prism 5 software (GraphPad Software, Inc.) was used for statistical analysis.

\section{Results}

DHA preserves articular cartilage in ACLT mice. Safranin O-fast green staining indicated that there was significant loss of proteoglycans, with OARSI scores increasing significantly in the ACLT+vehicle group relative to the sham-operated group at 4- and 8-weeks following surgery (Fig. 2A and D). Similarly, results of immunohistochemical staining demonstrated that the expression of MMP-13 (Fig. 2B and E) and VEGF (Fig. 2C and F) was significantly increased after 4 and 8 weeks. Administration of DHA resulted in retention of proteoglycans and OARSI scores that were improved in the ACLT+DHA group compared with the ACLT+vehicle group after 4 and 8 weeks (Fig. 2A and D). Aberrantly expressed MMP-13 (Fig. 2B and E) and VEGF (Fig. 2C and F) were recovered in the ACLT+DHA group compared with the ACLT+vehicle group at both 4- and 8-weeks following surgery.

DHA restores the microarchitecture of the subchondral bone in ACLT mice. Abnormal bone remodeling destroys the microstructure of subchondral bone which results in articular cartilage degeneration (21). It was investigated whether DHA was able to protect articular cartilage by preserving the microstructure of subchondral bone. The tibial subchondral bone structure of the tibia was analyzed by micro-CT. The results demonstrated that DHA improved the subchondral bone microstructure (Fig. 3A). BV/TV (Fig. 3B) and BMD (Fig. 3D) were significantly decreased in the ACLT+vehicle group compared with the sham-operated group at both 4- and 8-weeks but DHA 


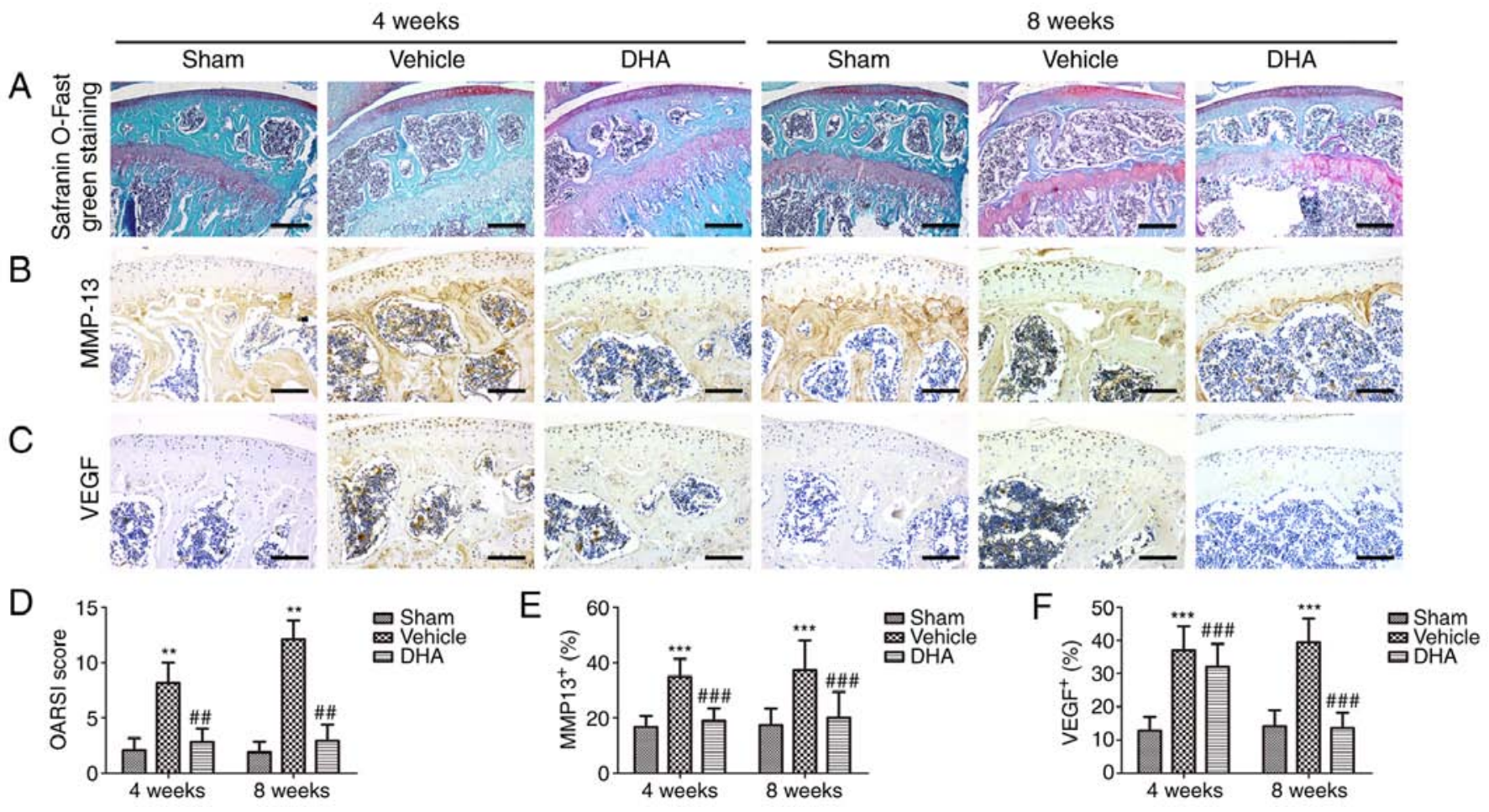

Figure 2. DHA preserves the articular cartilage in ACLT mice. (A) Histological analysis of articular cartilage using safranin O-fast green staining of sagittal sections of the medial compartment of the tibia. Scale bar, $200 \mu \mathrm{m}$. (B, C, E and F) Measurement of matrix (B and E) MMP-13 and (C and F) VEGF expression by immunohistochemical staining and quantitative analysis. Scale bar, $100 \mu \mathrm{m}$. (D) OARSI-modified Mankin score of articular cartilage at various time-points. Sham, sham-operated group; Vehicle, ACLT+vehicle group; DHA, ACLT+DHA group. $\mathrm{n}=10$ per group. ${ }^{* *} \mathrm{P}<0.01$ and ${ }^{* * * *} \mathrm{P}<0.001$, compared with the the sham-operated group. ${ }^{\# \#} \mathrm{P}<0.01$ and ${ }^{\# \# \#} \mathrm{P}<0.001$, compared with the ACLT + vehicle group. DHA, dihydroartemisinin; ACLT, anterior cruciate ligament transection; MMP-13, metalloproteinase-13; VEGF, vascular endothelial growth factor; OARSI, Osteoarthritis Research Society International.

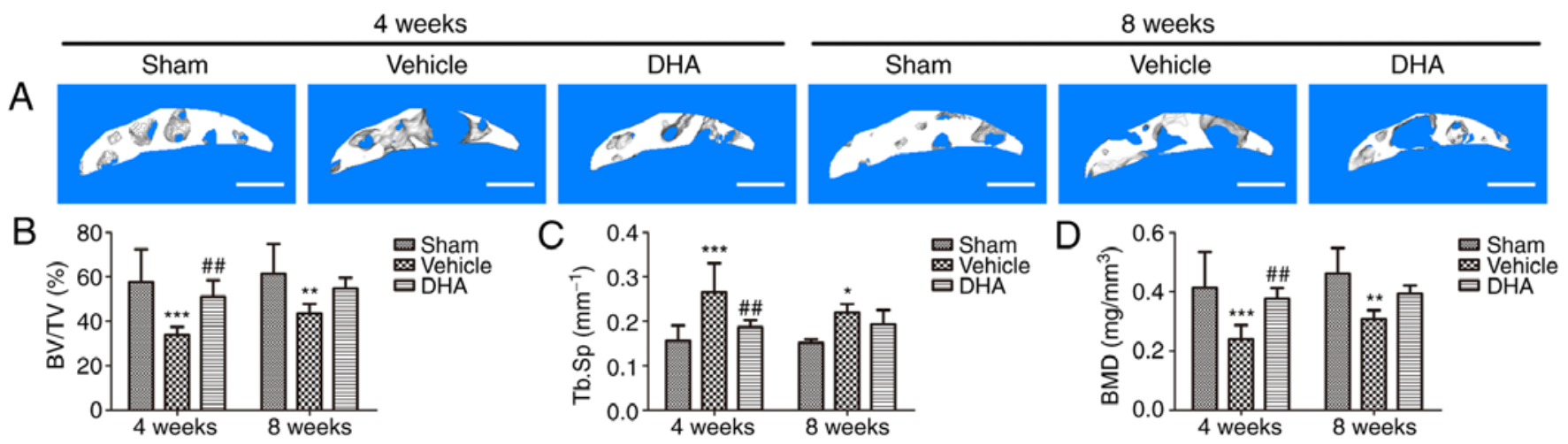

Figure 3. DHA restores the microarchitecture of the subchondral bone of ACLT mice. (A) 3D micro-CT reconstruction of sagittal views of the medial compartment of tibial subchondral bone at different time-points after sham or ACLT surgery. Scale bar, $500 \mu \mathrm{m}$. (B and D) Quantitative micro-CT analyses of the microarchitecture of tibial subchondral bone: (B) BV/TV (\%), (C) Tb.Sp and (D) BMD. Sham, sham-operated group; Vehicle, ACLT+vehicle group; DHA, ACLT+DHA group. $\mathrm{n}=6$ per group. ${ }^{*} \mathrm{P}<0.05,{ }^{* *} \mathrm{P}<0.01$ and ${ }^{* * *} \mathrm{P}<0.001$, compared with the sham-operated group. ${ }^{\# /} \mathrm{P}<0.01$, compared with the vehicle group. DHA, dihydroartemisinin; ACLT, anterior cruciate ligament transection; micro-CT, micro-computed tomography; BV/TV, bone volume/tissue volume; Tb.Sp, trabecular separation; BMD, bone mineral density.

abrogated these changes (ACLT+DHA group) relative to the ACLT+vehicle group 4-weeks after surgery (Fig. 3B and D). The Tb.Sp was significantly increased in the ACLT+vehicle group relative to the sham-operated group at both 4- and 8-weeks (Fig. 3C). DHA recovered these changes (ACLT+DHA group) compared with the ACLT+vehicle group 4-weeks after surgery (Fig. 3C). Collectively, these data indicated that systemic administration of DHA inhibited abnormal bone remodeling and restored the subchondral bone microstructure.
DHA inhibits abnormal bone remodeling in the subchondral bone in ACLT mice. Immunohistochemistry and immunofluorescence were performed to investigate whether systemic DHA was able to inhibit abnormal bone remodeling and angiogenesis in subchondral bone 4-weeks after surgery. RANKL-immunostained sections demonstrated a significant increase in the number of cells positive for RANKL in the ACLT+vehicle group relative to the sham-operated group (Fig. 4A and E). Administration of DHA significantly reduced 
A
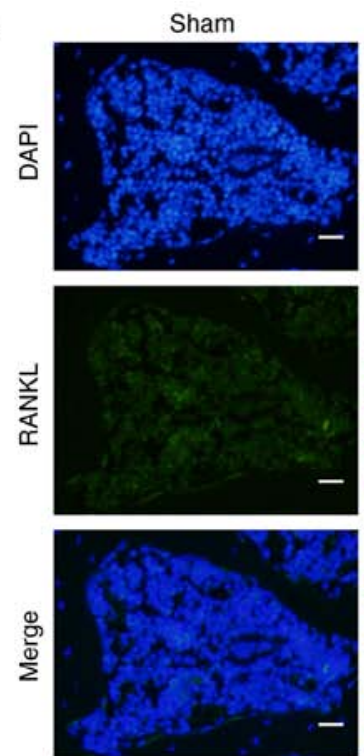

B

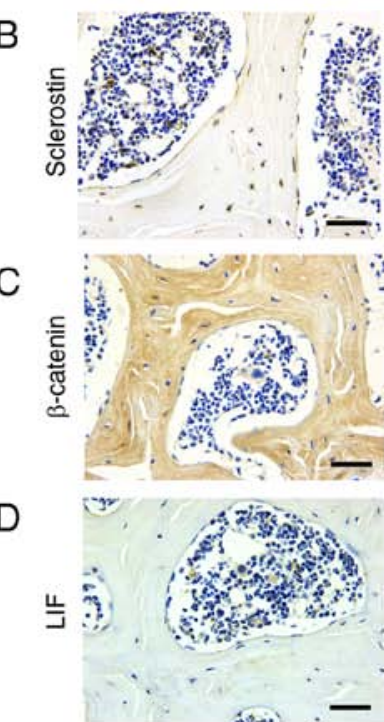

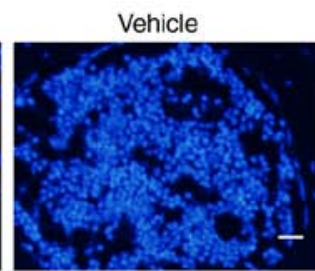
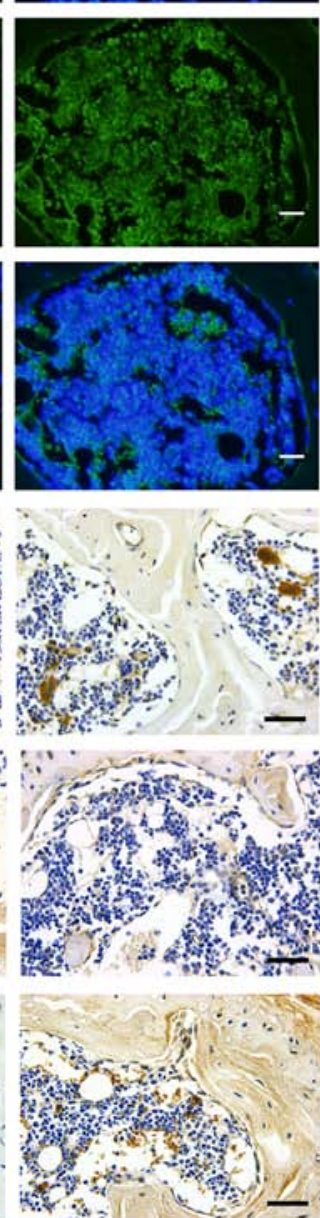
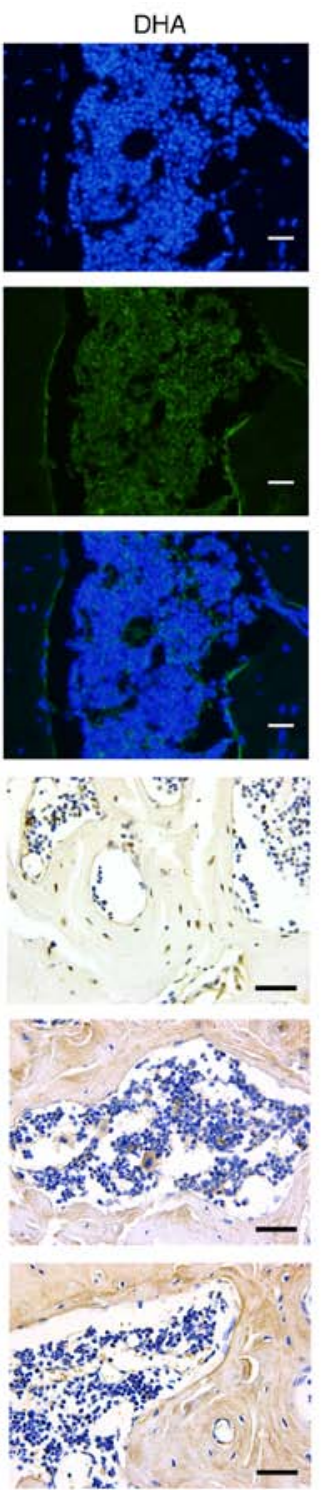

$\mathrm{E}$
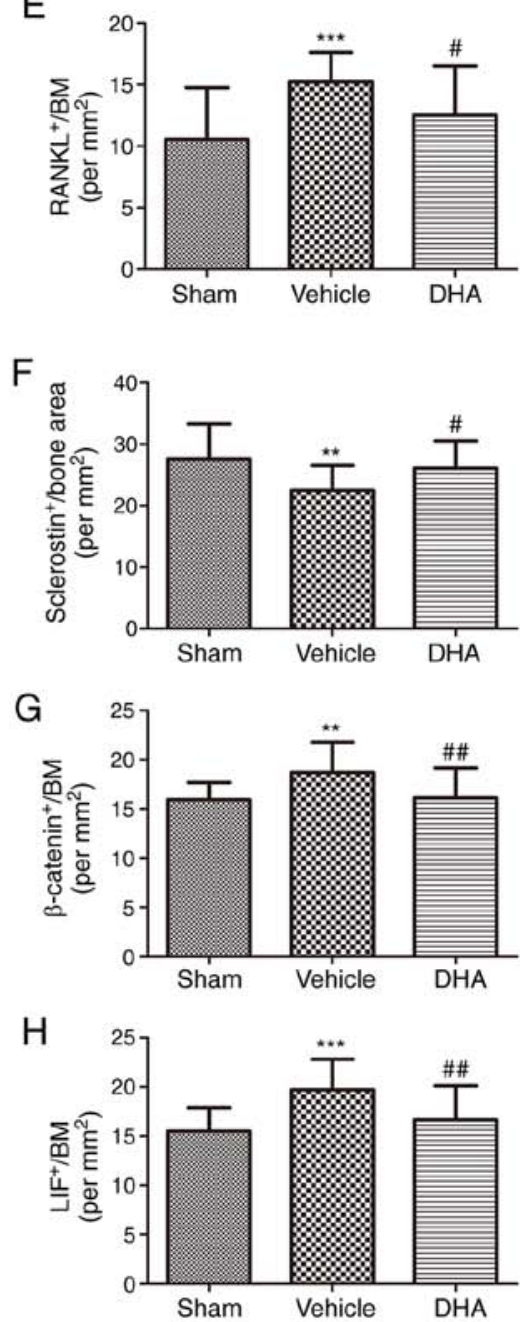

Figure 4. DHA inhibits abnormal bone remodeling in the subchondral bone of ACLT mice. (A and E) Immunofluorescence staining and quantification of the expression of RANKL (green) in tibial subchondral bone. Cell nuclei were stained blue using DAPI. Scale bar, $50 \mu \mathrm{m}$. (B-D and F-H) Immunohistochemical staining and quantification of the expression of (B and F) sclerostin, (C and G) $\beta$-catenin and (D and H) LIF in tibial subchondral bone. Scale bar, $50 \mu \mathrm{m}$. Sham, sham-operated group; Vehicle, ACLT+vehicle group; DHA, ACLT+DHA group. $\mathrm{n}=10$ per group. ${ }^{* *} \mathrm{P}<0.01$ and ${ }^{* * *} \mathrm{P}<0.001$, compared with the sham-operated group. ${ }^{\#} \mathrm{P}<0.05$ and ${ }^{\# \#} \mathrm{P}<0.01$, compared with the vehicle group. DHA, dihydroartemisinin; ACLT, anterior cruciate ligament transection; RANKL, receptor activator of nuclear factor $\kappa \mathrm{B}$ ligand; LIF, leukemia inhibitory factor.

this increased number relative to the ACLT+vehicle group (Fig. 4A and E). To investigate whether osteoclasts altered the expression of sclerostin in osteocytes, sclerostin expression was measured by immunohistochemistry. The results demonstrated a significant decrease in sclerostin expression in the ACLT+vehicle group relative to the sham-operated group (Fig. 4B and F). Treatment with DHA significantly increased sclerostin expression relative to the ACLT+vehicle group (Fig. 4B and F). The expression of $\beta$-catenin in osteoblasts was assessed by immunohistochemistry and a significant increase was observed in the ACLT+vehicle group compared with the sham-operated group (Fig. 4C and G). Administration of DHA significantly reduced $\beta$-catenin expression compared with the ACLT+vehicle group (Fig. 4C and G).

It was also investigated whether DHA inhibited the excessive expression of LIF, which is secreted by osteoclasts in the subchondral bone, using immunohistochemistry 4-weeks after surgery. The results demonstrated that a significant increase in LIF expression was observed in the ACLT+vehicle group compared with the sham-operated group (Fig. 4D and H). DHA significantly reduced this expression relative to the ACLT+vehicle group (Fig. 4D and H).

These results indicated that DHA inhibited the excessive expression of LIF secreted by osteoclasts in ACLT mice, thus reducing inhibition of sclerostin and preventing subchondral bone remodeling, thereby restoring the subchondral bone microstructure and inhibiting articular cartilage degeneration.

DHA inhibits abnormal angiogenesis in the subchondral bone in ACLT mice. Abnormal angiogenesis in the subchondral bone is a pathological feature of OA (32). An angiogenic marker, CD31-positive endothelial progenitor cells (33), was 
A
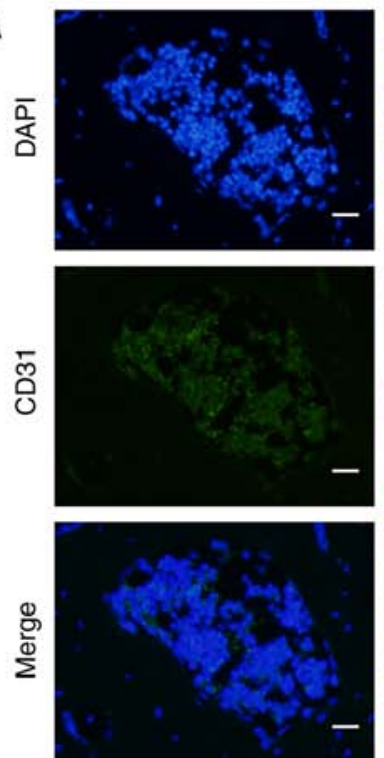
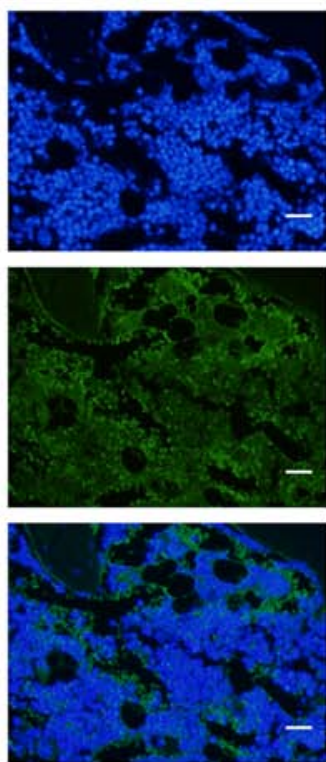
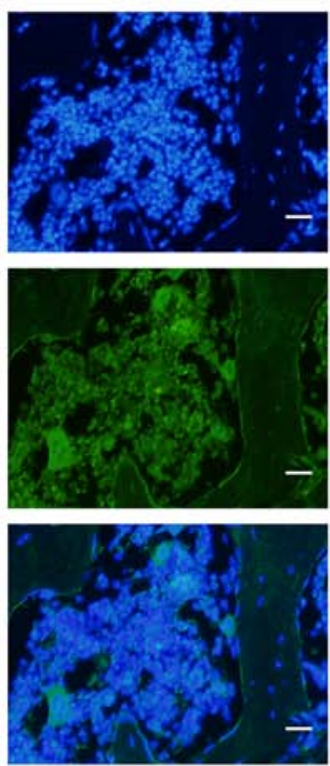

B

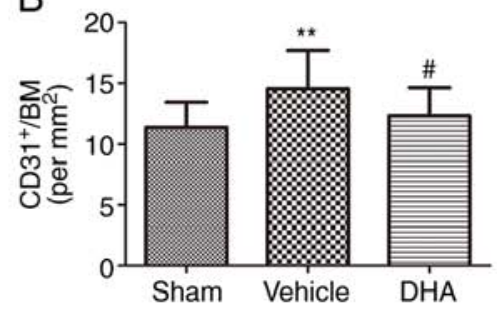

Figure 5. DHA inhibits abnormal angiogenesis in the subchondral bone of ACLT mice. (A and B) Immunofluorescence staining and quantification of the expression of CD31 (green) in tibial subchondral bone. Cell nuclei were stained blue using DAPI. Scale bar, $50 \mu \mathrm{m}$. Sham, sham-operated group; Vehicle, ACLT+vehicle group; DHA, ACLT+DHA group. $\mathrm{n}=10$ per group. ${ }^{* *} \mathrm{P}<0.01$, compared with the sham-operated group. ${ }^{*} \mathrm{P}<0.05$, compared with the vehicle group. DHA, dihydroartemisinin; ACLT, anterior cruciate ligament transection.

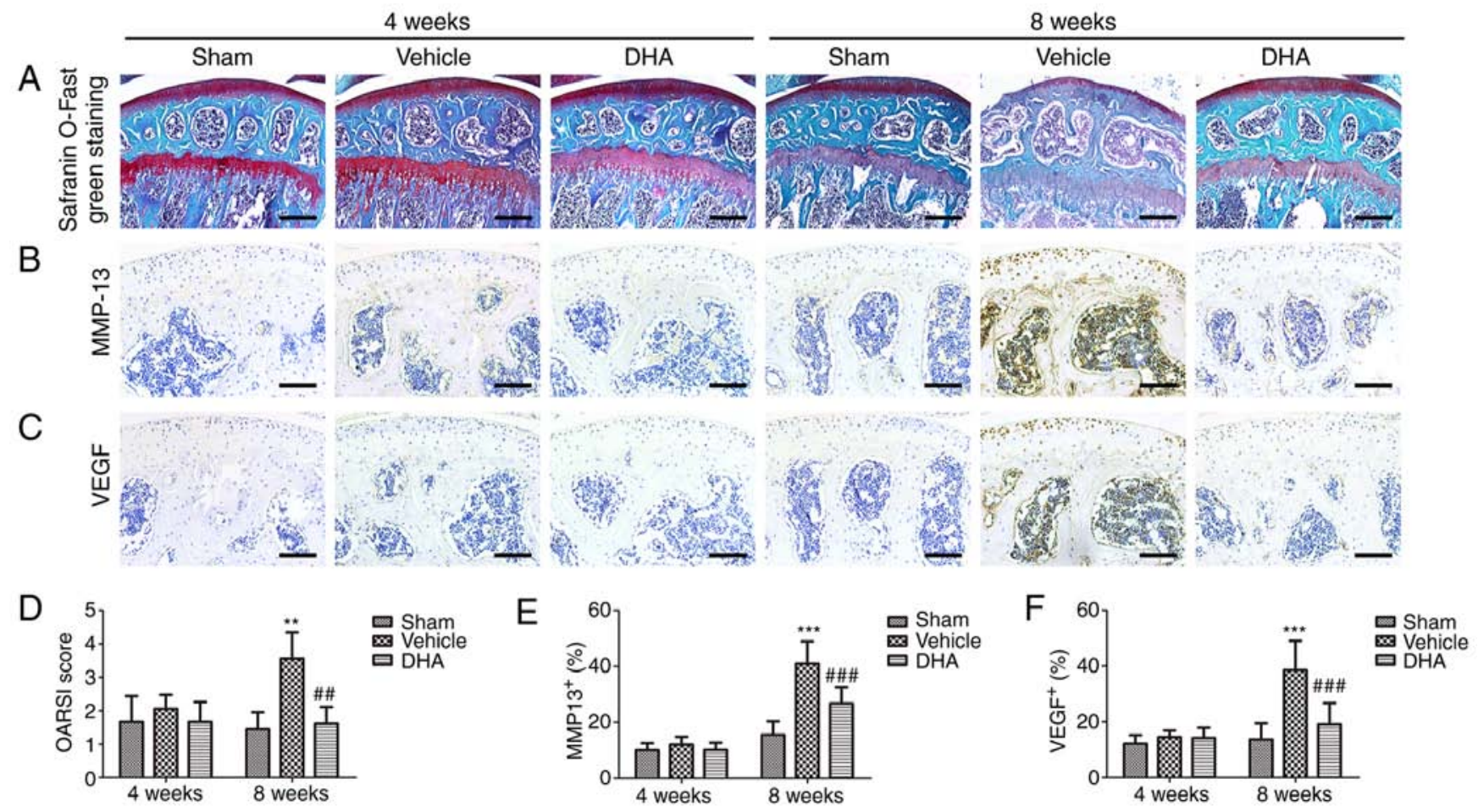

Figure 6. DHA preserves articular cartilage in OVX mice. (A) Histological analysis of articular cartilage using safranin O-fast green staining of sagittal sections of the medial compartment of the tibia. Scale bar, $200 \mu \mathrm{m}$. (B, C, E and F) Measurement of matrix (B and E) MMP-13 and (C and F) VEGF expression by immunohistochemical staining and quantitative analysis. Scale bar, $100 \mu \mathrm{m}$. (D) OARSI-modified Mankin score of articular cartilage at various time-points. Sham, sham-operated group; Vehicle, OVX+vehicle group; DHA, OVX+DHA group. $\mathrm{n}=10$ per group. ${ }^{* *} \mathrm{P}<0.01$ and ${ }^{* * * *} \mathrm{P}<0.001$, compared with the sham-operated group. ${ }^{\# \#} \mathrm{P}<0.01$ and ${ }^{\# \# "} \mathrm{P}<0.001$, compared with the OVX+vehicle group. DHA, dihydroartemisinin; OVX, ovariectomized; MMP-13, metalloproteinase-13; VEGF, vascular endothelial growth factor; OARSI, Osteoarthritis Research Society International.

assessed using immunofluorescence. The results indicated that its number significantly increased in the ACLT+vehicle group relative to the sham-operated group (Fig. 5) and that the number was reduced in the ACLT+DHA group compared with the ACLT+vehicle group (Fig. 5).
DHA preserves the articular cartilage in OVX mice. Safranin O-fast green staining indicated that there was significant loss of proteoglycans, with OARSI scores significantly increasing in the OVX+vehicle group relative to the sham-operated group at 8-weeks following surgery (Fig. 6A and D), but not 4-weeks. 
A

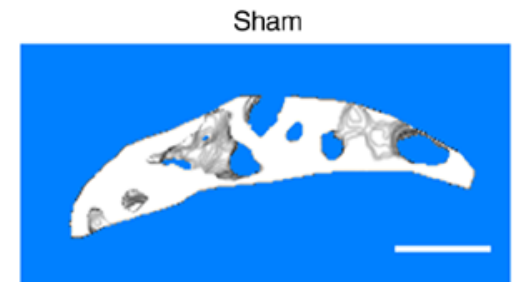

B

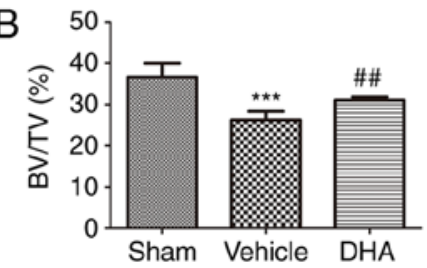

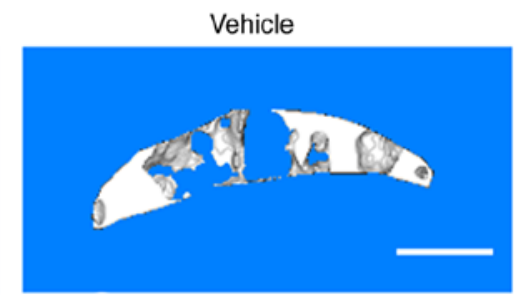

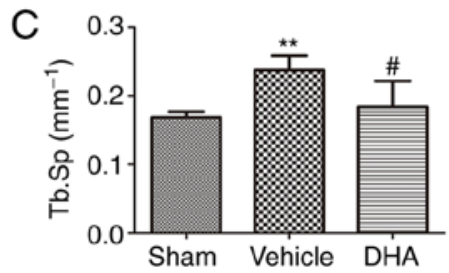

DHA

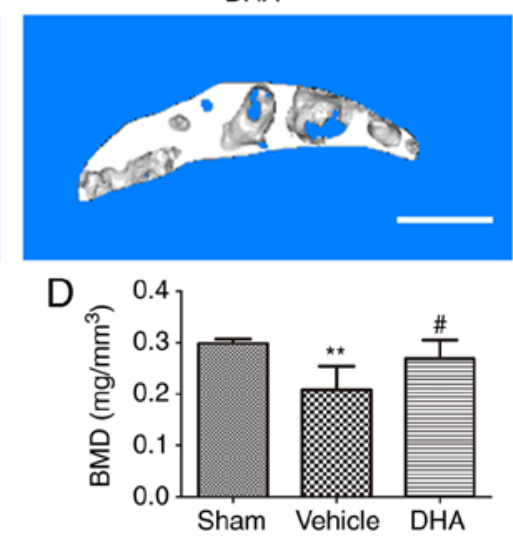

Figure 7. DHA restores the microarchitecture of the subchondral bone in OVX mice. (A) 3D micro-CT reconstruction of sagittal views of the medial compartment of tibial subchondral bone at different time-points after sham or OVX surgery. Scale bar, $500 \mu \mathrm{m}$. (B-D) Quantitative micro-CT analyses of the microarchitecture of tibial subchondral bone: (B) BV/TV (\%), (C) Tb.Sp and (D) BMD. Sham, sham-operated group; Vehicle, OVX+vehicle group; DHA, OVX+DHA group. $\mathrm{n}=6$ per group. ${ }^{* * *} \mathrm{P}<0.01$ and ${ }^{* * *} \mathrm{P}<0.001$, compared with the sham-operated group. ${ }^{\#} \mathrm{P}<0.05$ and ${ }^{\# \#} \mathrm{P}<0.01$, compared with the vehicle group. DHA, dihydroartemisinin; OVX, ovariectomized; micro-CT, micro-computed tomography; BV/TV, bone volume/tissue volume; Tb.Sp, trabecular separation; BMD, bone mineral density.

A
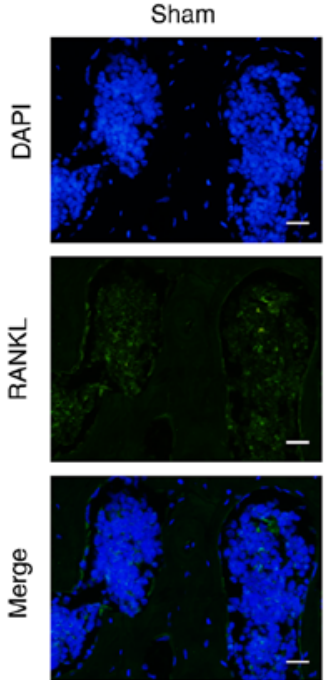

B
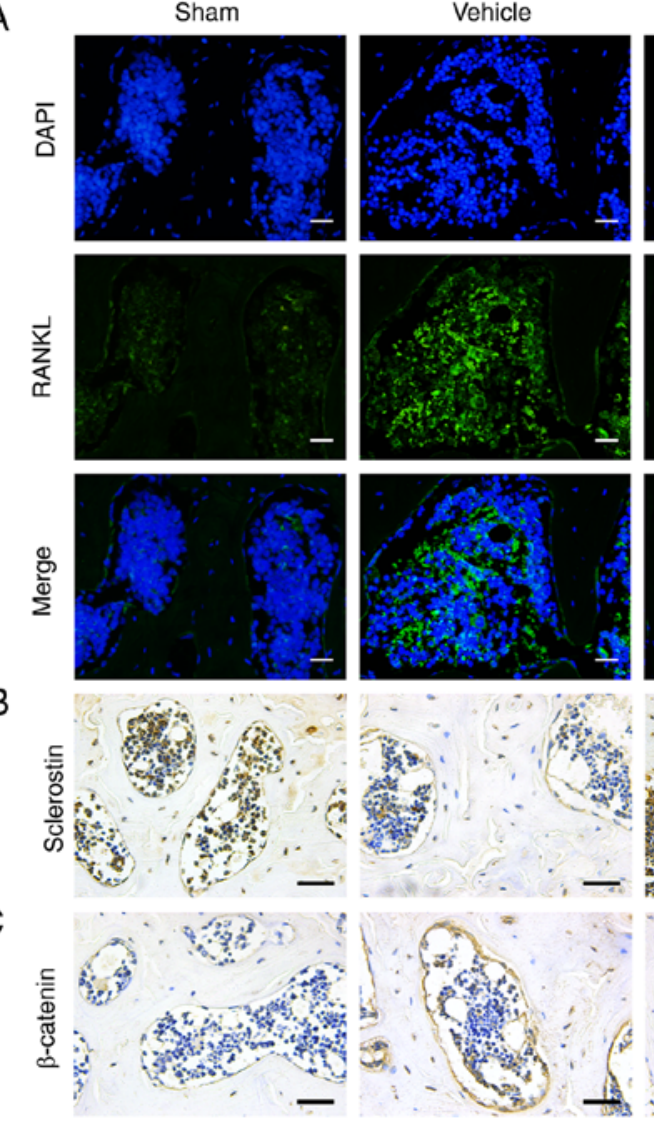

D

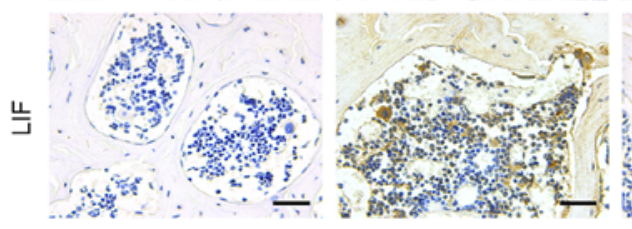

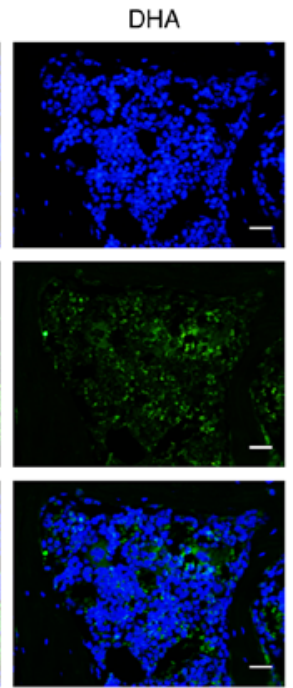
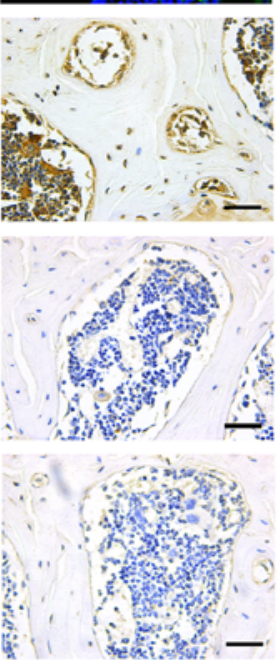

$\mathrm{E}$
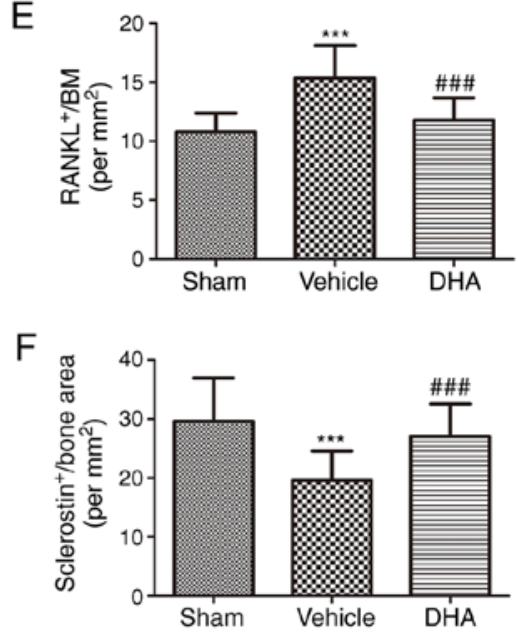

G
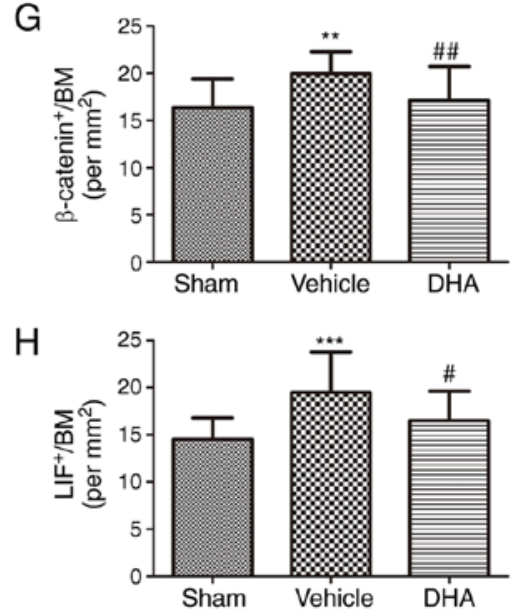

Figure 8. DHA inhibits abnormal bone remodeling in the subchondral bone of OVX mice. (A and E) Immunofluorescence staining and quantification of the expression of RANKL (green) in tibial subchondral bone. Cell nuclei were stained blue using DAPI. Scale bar, $50 \mu \mathrm{m}$. (B-D and F-H) Immunohistochemical staining and quantification of the expression of (B and F) sclerostin, $(\mathrm{C}$ and $\mathrm{G}) \beta$-catenin and (D and H) LIF in tibial subchondral bone. Scale bar, $50 \mu \mathrm{m}$. Sham, sham-operated group. Vehicle, OVX+vehicle group. DHA, OVX+DHA group. $\mathrm{n}=10$ per group. ${ }^{* * *} \mathrm{P}<0.01$ and ${ }^{* * * *} \mathrm{P}<0.001$, compared with the sham-operated group. ${ }^{\#} \mathrm{P}<0.05$ and ${ }^{\# \#} \mathrm{P}<0.01$ and ${ }^{\# \# \#} \mathrm{P}<0.001$ compared with the vehicle group. DHA, dihydroartemisinin; OVX, ovariectomized; RANKL, receptor activator of nuclear factor $\kappa \mathrm{B}$ ligand; LIF, leukemia inhibitory factor. 

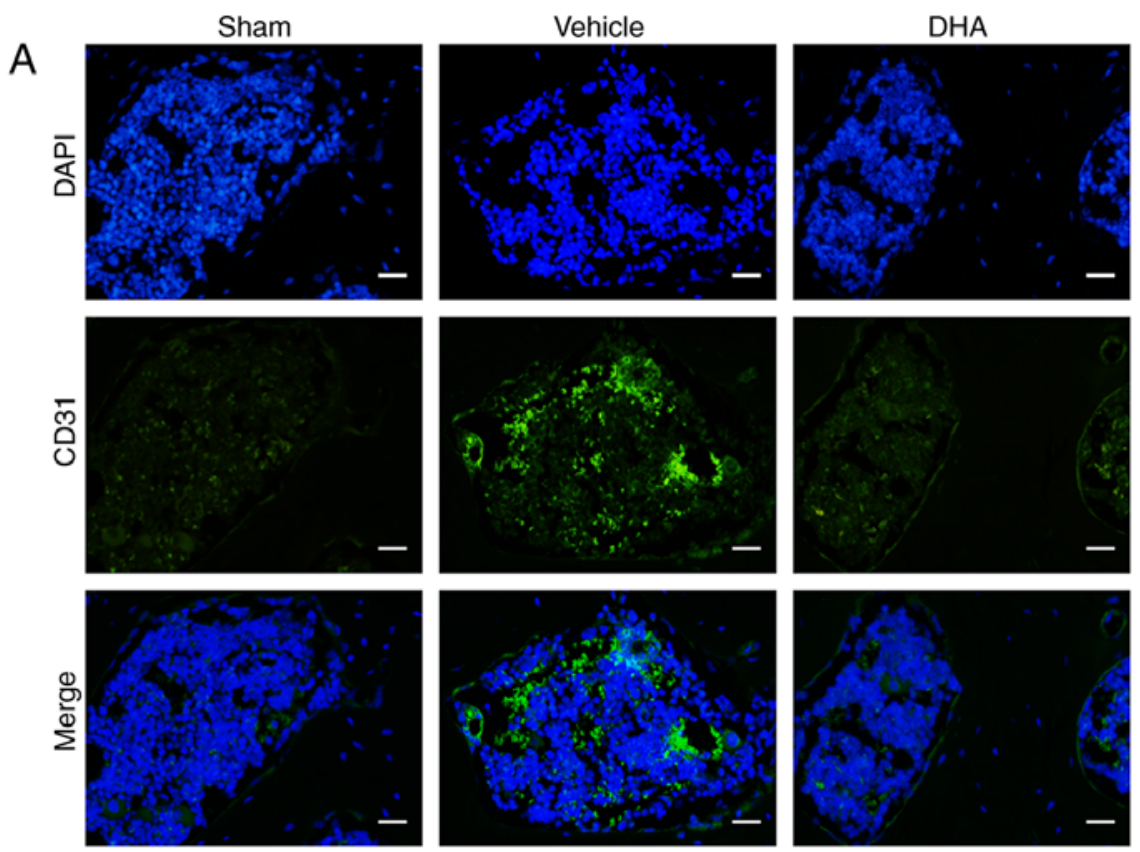

B

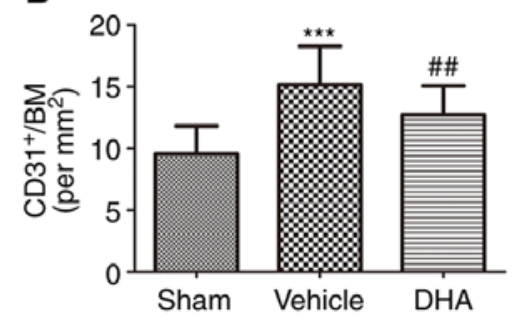

Figure 9. DHA inhibits abnormal angiogenesis in the subchondral bone of OVX mice. (A and B) Immunofluorescence staining and quantification of the expression of CD31 (green) in tibial subchondral bone. Cell nuclei were stained blue using DAPI. Scale bar, $50 \mu \mathrm{m}$. Sham, sham-operated group; Vehicle, OVX+vehicle group; DHA, OVX+DHA group. $\mathrm{n}=10$ per group. ${ }^{* * *} \mathrm{P}<0.001$, compared with the sham-operated group. ${ }^{\# \#} \mathrm{P}<0.01$, compared with the vehicle group.

Similarly, the results of the immunohistochemical staining demonstrated that the expression of MMP-13 (Fig. 6B and E) and VEGF (Fig. 6C and F) significantly increased after 8-weeks, but not 4-weeks. Administration of DHA resulted in retention of proteoglycans and OARSI scores that were improved in the OVX+DHA group compared with the OVX+vehicle group after 8-weeks (Fig. 6A and D). Aberrantly expressed MMP-13 (Fig. 6B and E) and VEGF (Fig. 6C and F) were recovered in the OVX+DHA group compared with the OVX+vehicle group at 8-weeks following surgery.

DHA restores the microarchitecture of the subchondral bone in OVX mice. Abnormal bone remodeling destroys the microstructure of the subchondral bone which results in articular cartilage degeneration (21). It was investigated whether DHA was able to protect the articular cartilage by preserving the microstructure of the subchondral bone. The tibial subchondral bone structure of the tibia was analyzed by micro-CT. The mouse OA model established by OVX, was established 8-weeks after surgery, but not 4-weeks. The OVX mice were also euthanized after 4 weeks but model establishment was not successful at that time-point. Therefore, the micro-CT result only contains 8 -week results. The results demonstrated that DHA improved the subchondral bone microstructure (Fig. 7A). BV/TV (Fig. 7B) and BMD (Fig. 7D) significantly decreased in the OVX+vehicle group compared with the sham-operated group at 8-weeks, but DHA abrogated these changes (OVX+DHA group) relative to the OVX+vehicle group 8-weeks after surgery (Fig. 7B and D). The Tb.Sp significantly increased in the OVX+vehicle group relative to the sham-operated group at 8-weeks (Fig. 7C). DHA recovered these changes (OVX+DHA group) compared with the OVX+vehicle group 8-weeks after surgery (Fig. 7C). Collectively, these data indicated that systemic administration of DHA inhibited abnormal bone remodeling and restored subchondral bone microstructure.

DHA inhibits abnormal bone remodeling in subchondral bone in OVX mice. Immunohistochemistry and immunofluorescence were performed to investigate whether systemic DHA was able to inhibit abnormal bone remodeling and angiogenesis in the subchondral bone 8-weeks after surgery. RANKL-immunostained sections demonstrated a significant increase in the number of cells positive for RANKL in the $\mathrm{OVX}+$ vehicle group relative to the sham-operated group (Fig. 8A and E). Administration of DHA significantly reduced that increased number relative to the OVX+vehicle group (Fig. 8A and E). To investigate whether osteoclasts altered the expression of sclerostin in osteocytes, sclerostin expression was assessed by immunohistochemistry. The results demonstrated a significant decrease in sclerostin expression in the OVX+vehicle group relative to the sham-operated group (Fig. 8B and F). Treatment with DHA significantly increased sclerostin expression relative to the OVX+vehicle group (Fig. 8B and F). The expression of $\beta$-catenin in osteoblasts was assessed by immunohistochemistry and a significant increase was observed in the OVX+vehicle group compared with the sham-operated group (Fig. 8C and G). Administration of DHA significantly reduced $\beta$-catenin expression compared with the OVX+vehicle group (Fig. 8C and G).

It was investigated whether DHA inhibited excessive expression of LIF secreted by osteoclasts in the subchondral bone using immunohistochemistry 8 -weeks after surgery. The results demonstrated that a significant increase in LIF expression was observed in the OVX+vehicle group compared with sham-operated group (Fig. 8D and H). DHA significantly reduced this expression relative to the OVX+vehicle group (Fig. 8D and H). 
The results indicated that DHA inhibited excessive expression of LIF secreted by osteoclasts in OVX mice, thus reducing inhibition of sclerostin and preventing subchondral bone remodeling, thereby restoring the subchondral bone microstructure and inhibiting articular cartilage degeneration.

DHA inhibits abnormal angiogenesis in subchondral bone in OVX mice. Abnormal angiogenesis in subchondral bone is a pathological feature of OA (32). The number of CD31-positive endothelial progenitor cells were assessed using immunofluorescence. The results indicated that compared with the sham-operated group, their numbers significantly increased in the OVX+vehicle group (Fig. 9) and that compared with the OVX+vehicle group, their numbers were decreased in the OVX+DHA group (Fig. 9).

\section{Discussion}

$\mathrm{OA}$ is the most common degenerative joint disease and possesses a complex pathogenesis. Abnormal bone remodeling and angiogenesis in the subchondral bone destroys the subchondral bone microstructure and leads to articular cartilage degeneration $(22,23)$. The present study revealed that DHA decreased the inhibition of sclerostin by reducing the LIF secretion of osteoclasts and, hence, attenuated aberrant bone remodeling and inhibited angiogenesis in subchondral bone, thereby preserving the subchondral bone microstructure and attenuating articular cartilage degeneration.

The microstructural integrity of the subchondral bone is especially important for the protection of articular cartilage (2). Studies have revealed that microstructural changes in subchondral bone may precede the degeneration in articular cartilage in OA $(3,4)$. The integrity of the bone microstructure is maintained through the coupling of bone remodeling, which includes the temporal and spatial balance of bone resorption and formation. This in turn involves resorption of the bone matrix by osteoclasts, normally replaced by new bone matrix by osteoblasts (34). Abnormal bone remodeling can disrupt the homeostatic balance of the bone and destroy the integrity of the bone microstructure (35). At present, the mechanisms that balance bone resorption and formation resulting in bone remodeling remain unclear.

The most commonly used surgical method of inducing OA is ACLT. In this method, the ACL is transected with micro-scissors, which causes joint destabilization. The anterior drawer test with the joint flexed is used to confirm that transection of the ligament has occurred. The ACLT leads to the increase of joint mechanical stress. The increased load on the posterior tibial plateau leads to the destruction of cartilage and subchondral bone, which leads to OA $(29,36)$. Osteoclasts are the sole cells that resorb bone matrix, through dissolution by the secretion of acids and proteases. RANKL is a key factor in osteoclastogenesis (37). The secretion of RANKL by osteoblast precursors following abnormal mechanical stimulation results in its binding to RANK on osteoclast precursors, inducing osteoclastogenesis that mediates bone resorption (22). The prevalence of OA in postmenopausal women is higher than that in men. Ovariectomy significantly reduced the production of estrogen in mice, resulting in increased bone resorption mediated by subchondral bone osteoclasts, resulting in increased subchondral bone loss and bone microstructure deterioration, thereby promoting articular cartilage degeneration and inducing osteoarthritis $(31,38)$. Osteoporosis (OP) is characterized by increased bone loss, deterioration of bone microarchitecture and an increased risk of fragility fractures (39). The pathogenesis of OA has not been fully elucidated. OA is considered a whole-joint disease in which all components are involved, with the subchondral bone, which is located below the articular cartilage, playing a significant role in disease pathogenesis (40). In the subchondral bone of OA, osteoclast-mediated bone resorption increases bone loss, and its microstructure deteriorates, which leads to articular cartilage degeneration (41). Excessive bone resorption, which is a hallmark of postmenopausal OP, also occurs at the early stage in the development of OA (42). They both have increased bone remodeling, increased osteoclast-mediated bone resorption, and deterioration of bone microstructure in OVX mice. The present study demonstrated that the expression of RANKL significantly increased in the ACLT+vehicle and OVX+vehicle group compared with the sham-operated group. This indicates that RANKL-induced osteoclastogenesis is significantly enhanced in ACLT mice. Consistent with previous studies, the present results revealed that DHA reduced bone resorption by inhibiting RANKL-mediated osteoclastogenesis.

The Wnt/ $\beta$-catenin signaling pathway is considered the most important pathway that regulates bone homeostasis (43). Activation of the Wnt/ $\beta$-catenin pathway promotes differentiation of bone marrow mesenchymal stem cells into osteoblasts, promoting osteoblast proliferation and maturation, inhibiting osteoblast apoptosis, and thereby promoting osteoblast-mediated bone formation (44). Sclerostin, encoded by the SOST gene, is secreted by osteocytes and binds to LDL receptor-related protein (LRP) $5 / 6(13,45)$. It is an antagonist of the Wnt/ $\beta$-catenin signalling (13). Sclerostin inhibits osteoblast-mediated bone formation by binding to LRP5/6 on osteoblasts (45). Previous studies have revealed that SOST knockout (KO) mice and postmenopausal osteoporotic rats treated with sclerostin antibodies exhibited a significant increase in bone volume, bone formation, and the number of osteoblasts on the surface of their bones (46). As OA progresses, sclerostin expression significantly decreases, causing $\beta$-catenin expression to significantly increase in the subchondral bone leading to gradually worsening articular cartilage degeneration in humans (47). The present study demonstrated that compared with the sham-operated group, sclerostin expression in osteocytes significantly decreased and $\beta$-catenin expression in osteoblasts significantly increased in the subchondral bone in the ACLT+vehicle group. This indicated that decreased expression of sclerostin in the subchondral bone caused abnormal remodeling that destroyed its microstructural integrity in ACLT mice. After OVX, OP caused by oestrogen revealed an increase in bone remodeling, specifically osteoclast-mediated enhancement of bone resorption, and osteoblast-mediated bone formation was relatively weakened (9). In OVX-induced OP-related osteoarthritis, subchondral bone remodeling increases, characterized by increased bone loss, decreased bone mass, decreased bone density, and deteriorated bone microarchitecture $(9,12,31)$. The present study also demonstrated that compared with the sham-operated group, sclerostin expression significantly 
decreased in osteocytes, and $\beta$-catenin expression also significantly increased in osteoblasts in the subchondral bone in the OVX+vehicle group. This indicated that the decreased expression of sclerostin in the subchondral bone of OP-induced mouse OA caused abnormal bone formation and abnormality of the subchondral bone remodeling, which destroyed the microstructural integrity of the subchondral bone.

LIF is a bone remodeling regulatory protein that is secreted by osteoclasts and binds to a receptor complex of glycoprotein 130 (gp130)/LIF receptor (LIFR) on osteocytes, which inhibits sclerostin $(48,49)$. Thus, the inhibitory effect of sclerostin on Wnt/ $\beta$-catenin in osteoblasts is reduced, promoting osteoblast-mediated bone formation in RANKL transgenic mice and OPG-KO mice compared with wild-type mice (20). Treatment of OPG-KO mice with anti-RANKL antibody, an anti-bone resorption agent, was revealed to suppress the expression of LIF and increase the expression of sclerostin, thereby reducing bone formation via inhibition of the Wnt/ $\beta$-catenin pathway (20). Specific knockout of gp130 in osteocytes was revealed to increase the expression of sclerostin, and, thus, inhibit bone formation in mice (50). The aforementioned studies demonstrated that the osteoclast-derived factor LIF inhibited the expression of sclerostin in osteocytes and, hence, promoted bone formation. The present study demonstrated that the expression of LIF in osteoclasts significantly increased in the ACLT+vehicle group relative to the sham-operated group. We inhibited osteoclastogenesis by DHA which reduced the expression of the osteoclast-derived factor LIF, thereby increasing the expression of sclerostin that inhibits abnormal bone formation and remodeling of the subchondral bone. Sclerostin is an important factor in bone remodeling, and is affected by numerous factors $(51,52)$. Previous studies have found that mechanical stimulation inhibit the expression of sclerostin (16-18). Studies on osteoporosis have revealed that osteoclasts oversecrete LIF and inhibit the expression of sclerostin (20); however, in the subchondral bone of OA, its mechanism is unclear. We first established a model of OA in mice with ACLT mechanical injury and it was revealed that LIF secreted by osteoclasts decreased the expression of sclerostin. However, the influence of the increased mechanical stress in the ACLT model cannot be completely eliminated. We then used the OVX-induced OP-related mouse OA model to further investigate the effect of LIF secreted by osteoclasts on sclerostin. The present study revealed a significant increase in the expression of LIF in osteoclasts in the OVX+vehicle group compared with that in the sham-operated group. After treatment with DHA, the expression of LIF in OVX+DHA group was significantly lower than that in OVX+vehicle group. This indicated that DHA inhibited osteoclastogenesis to reduce the expression of osteoclast-derived factor LIF, and, therefore, the expression of sclerostin was significantly increased. Compared with the OVX+vehicle group, DHA significantly decreased Tb.Sp and significantly increased BV/TV (\%) and BMD in the subchondral bone of the OVX+DHA group. Similarly, the expression of MMP-13 and VEGF and the OARSI score in the articular cartilage were significantly decreased. This indicated that DHA reduced the expression of osteoclast-derived factor LIF by inhibiting osteoclastogenesis, thereby reducing the inhibition of bone sclerotin by LIF, reducing abnormal bone formation, inhibiting the increase of subchondral bone remodeling, and retaining the microstructure of the subchondral bone to slow the degeneration of articular cartilage.

Angiogenesis provides oxygen and nutrients for bone formation, which is associated with bone remodeling for bone homeostasis (50). However, abnormal angiogenesis is a key pathological feature of OA in the subchondral bone. Abnormal angiogenesis in subchondral bone increases with increased bone remodeling, which leads to abnormal bone formation $(22,23)$. CD31, encoded by the platelet endothelial cell adhesion molecule (PECAM1) gene, is a specific marker of endothelial progenitor cells and is used to assess angiogenesis (32). The present study demonstrated that CD31-positive endothelial progenitor cells in the subchondral bone significantly increased in number in the ACLT+vehicle group. Previous studies have also revealed that DHA has anti-angiogenic effects $(27,53,54)$. After treatment with DHA, CD31-positive staining significantly decreased in the ACLT+DHA group relative to the ACLT+vehicle group. Previous tudies have revealed that in the OA model of OVX rats, the expression of CD31 in the subchondral bone of the model group was significantly increased, and the microstructural destruction of the subchondral bone similarly with the expression of MMP-13 in the articular cartilage was increased, and the loss of proteoglycan was severe $(9,12,55)$. The present study revealed a significant increase in the number of CD31-positively labeled endothelial progenitor cells in the subchondral bone of the OVX+vehicle group compared with sham-operated group. However, the role of DHA in OVX-induced abnormal angiogenesis of the subchondral bone in OP-associated OA remains unclear. The present experiments revealed that CD31 expression was significantly reduced in the $\mathrm{OVX}+\mathrm{DHA}$ group compared with the OVX+vehicle group. These results indicated that DHA inhibited abnormal angiogenesis of subchondral bone and slowed abnormal bone remodeling.

DHA is an effective drug against malaria, and a semi-synthetic derivative of artemisinin with fewer side effects. It performs an important role in inhibiting inflammation, is anti-angiogenic, suppresses cancer and is anti-osteoclastogenic (26-28). DHA suppresses the expression of osteoclast marker genes, such as cathepsin K, calcitonin receptor and tartrate resistant acid phosphatase (TRACP); DHA also inhibits RANKL-induced osteoclast formation and bone resorption, thus reversing the ovariectomized bone loss in oestrogen deficient-induced osteoporosis of OVX mice (27). In addition, DHA effectively inhibited osteoclastogenesis and prevented breast cancer-induced osteolysis (28). Abnormal bone remodeling and angiogenesis in the subchondral bone destroys its microstructure and causes articular cartilage degeneration $(22,23)$. The present study revealed that DHA reduced the expression of the osteoclast-derived factor LIF by inhibiting osteoclastogenesis, thereby reducing inhibition of sclerostin and thus suppressing abnormal subchondral bone remodeling. In addition, DHA inhibited angiogenesis resulting in less abnormal bone remodeling. Therefore, DHA attenuated articular cartilage degeneration by inhibiting abnormal bone remodeling and angiogenesis in OA mice.

In conclusion, DHA decreased the inhibition of sclerostin by reducing LIF secretion by osteoclasts and thus attenuated aberrant bone remodeling and inhibited angiogenesis in the subchondral bone, thereby preserving the subchondral bone 
microstructure and attenuating articular cartilage degeneration. Future studies are required to further verify the underlying mechanism of DHA decreasing the inhibition of sclerostin by reducing LIF secretion by osteoclasts, such as anti-LIF antibodies.

\section{Acknowledgements}

Not applicable.

\section{Funding}

The present study was supported by the National Natural Science Foundation of China (grant nos. 81660373).

\section{Availability of data and materials}

The datasets used during the current study are available from the corresponding author on reasonable request.

\section{Authors' contributions}

QJ designed the experiment. LM, XZ, YL, JW and XY conducted the main experiments. LM analyzed the data, drafted the manuscript, and revised it. All the authors read and approved the final submitted manuscript.

\section{Ethics approval and consent to participate}

The experimental procedures were carried out in accordance with the National Institutes of Health Guide for the Care and Use of Laboratory Animals, and were approved by the Scientific Research Ethics Committee of the General Hospital of Ningxia Medical University (Yinchuan, China) (protocol no. 2016-147).

\section{Patient consent for publication}

Not applicable.

\section{Competing interests}

The authors declare that they have no competing interests.

\section{References}

1. Martel-Pelletier J, Barr AJ, Cicuttini FM, Conaghan PG, Cooper C, Goldring MB, Goldring SR, Jones G, Teichtahl AJ and Pelletier JP: Osteoarthritis. Nat Rev Dis Primers 2: 16072, 2016.

2. Goldring SR and Goldring MB: Changes in the osteochondral unit during osteoarthritis: Structure, function and cartilage-bone crosstalk. Nat Rev Rheumatol 12: 632-644, 2016.

3. Hugle $\mathrm{T}$ and Geurts J: What drives osteoarthritis?-synovial versus subchondral bone pathology. Rheumatology (Oxford) 56: 1461-1471, 2017.

4. Huebner JL, Hanes MA, Beekman B, TeKoppele JM and Kraus VB: A comparative analysis of bone and cartilage metabolism in two strains of guinea-pig with varying degrees of naturally occurring osteoarthritis. Osteoarthritis Cartilage 10: 758-767, 2002.

5. Eriksen EF: Cellular mechanisms of bone remodeling. Rev Endocr Metab Disord 11: 219-227, 2010.

6. Bailey AJ, Mansell JP, Sims TJ and Banse X: Biochemical and mechanical properties of subchondral bone in osteoarthritis. Biorheology 41: 349-358, 2004.
7. Radin EL and Rose RM: Role of subchondral bone in the initiation and progression of cartilage damage. Clin Orthop Relat Res: 34-40, 1986.

8. Bellido M, Lugo L, Roman-Blas JA, Castañeda S, Caeiro JR, Dapia S, Calvo E, Largo R and Herrero-Beaumont G: Subchondral bone microstructural damage by increased remodelling aggravates experimental osteoarthritis preceded by osteoporosis. Arthritis Res Ther 12: R152, 2010

9. Zhu S, Chen K, Lan Y, Zhang N, Jiang R and Hu J: Alendronate protects against articular cartilage erosion by inhibiting subchondral bone loss in ovariectomized rats. Bone 53: 340-349, 2013

10. Khorasani MS, Diko S, Hsia AW, Anderson MJ, Genetos DC, Haudenschild DR and Christiansen BA: Effect of alendronate on post-traumatic osteoarthritis induced by anterior cruciate ligament rupture in mice. Arthritis Res Ther 17: 30, 2015.

11. Miyauchi Y, Sato Y, Kobayashi T, Yoshida S, Mori T, Kanagawa H, Katsuyama E, Fujie A, Hao W, Miyamoto K, et al: HIFlalpha is required for osteoclast activation by estrogen deficiency in postmenopausal osteoporosis. Proc Natl Acad Sci USA 110: 16568-16573, 2013.

12. Cui Z, Xu C, Li X, Song J and Yu B: Treatment with recombinant lubricin attenuates osteoarthritis by positive feedback loop between articular cartilage and subchondral bone in ovariectomized rats. Bone 74: 37-47, 2015.

13. Li X, Zhang Y, Kang H, Liu W, Liu P, Zhang J, Harris SE and Wu D: Sclerostin binds to LRP5/6 and antagonizes canonical Wnt signaling. J Biol Chem 280: 19883-19887, 2005.

14. van Bezooijen RL, ten Dijke P, Papapoulos SE and Löwik CW: SOST/sclerostin, an osteocyte-derived negative regulator of bone formation. Cytokine Growth Factor Rev 16: 319-327, 2005.

15. Jia H, Ma X, Wei Y, Tong W, Tower RJ, Chandra A, Wang L, Sun Z, Yang Z, Badar F, et al: Loading-induced reduction in sclerostin as a mechanism of subchondral bone plate sclerosis in mouse knee joints during late-stage osteoarthritis. Arthritis Rheumatol 70: 230-241, 2018.

16. Robling AG, Niziolek PJ, Baldridge LA, Condon KW, Allen MR, Alam I, Mantila SM, Gluhak-Heinrich J, Bellido TM, Harris SE and Turner $\mathrm{CH}$ : Mechanical stimulation of bone in vivo reduces osteocyte expression of Sost/sclerostin. J Biol Chem 283: 5866-5875, 2008

17. Lin C, Jiang X, Dai Z, Guo X, Weng T, Wang J, Li Y, Feng G, Gao $X$ and He L: Sclerostin mediates bone response to mechanical unloading through antagonizing Wnt/beta-catenin signaling. J Bone Miner Res 24: 1651-1661, 2009.

18. Tu X, Rhee Y, Condon KW, Bivi N, Allen MR, Dwyer D, Stolina M, Turner CH, Robling AG, Plotkin LI and Bellido T: Sost downregulation and local Wnt signaling are required for the osteogenic response to mechanical loading. Bone 50: 209-217, 2012.

19. Sims NA and Johnson RW: Leukemia inhibitory factor: A paracrine mediator of bone metabolism. Growth Factors 30: 76-87, 2012.

20. Koide M, Kobayashi Y, Yamashita T, Uehara S, Nakamura M, Hiraoka BY, Ozaki Y, Iimura T, Yasuda H, Takahashi N and Udagawa N: Bone formation is coupled to resorption via suppression of sclerostin expression by osteoclasts. J Bone Miner Res 32: 2074-2086, 2017.

21. Burr DB and Gallant MA: Bone remodelling in osteoarthritis. Nat Rev Rheumatol 8: 665-673, 2012.

22. Cui Z, Crane J, Xie H, Jin X, Zhen G, Li C, Xie L, Wang L, Bian Q, Qiu T, et al: Halofuginone attenuates osteoarthritis by inhibition of TGF-beta activity and $\mathrm{H}$-type vessel formation in subchondral bone. Ann Rheum Dis 75: 1714-1721, 2016.

23. Zhen G, Wen C, Jia X, Li Y, Crane JL, Mears SC, Askin FB, Frassica FJ, Chang W, Yao J, et al: Inhibition of TGF-beta signaling in mesenchymal stem cells of subchondral bone attenuates osteoarthritis. Nat Med 19: 704-712, 2013.

24. Ji B, Zhang Z, Guo W, Ma H, Xu B, Mu W, Amat A and Cao L: Isoliquiritigenin blunts osteoarthritis by inhibition of bone resorption and angiogenesis in subchondral bone. Sci Rep 8: 1721,2018

25. Klayman DL: Qinghaosu (artemisinin): An antimalarial drug from China. Science 228: 1049-1055, 1985.

26. Tu Y: The development of new antimalarial drugs: Qinghaosu and dihydro-qinghaosu. Chin Med J (Engl) 112: 976-977, 1999.

27. Zhou L, Liu Q, Yang M, Wang T, Yao J, Cheng J, Yuan J, Lin X, Zhao J, Tickner J and Xu J: Dihydroartemisinin, an anti-malaria drug, suppresses estrogen deficiency-induced osteoporosis, osteoclast formation, and RANKL-induced signaling pathways. J Bone Miner Res 31: 964-974, 2016. 
28. Feng MX, Hong JX, Wang Q, Fan YY, Yuan CT, Lei XH, Zhu M, Qin A, Chen HX and Hong D: Dihydroartemisinin prevents breast cancer-induced osteolysis via inhibiting both breast caner cells and osteoclasts. Sci Rep 6: 19074, 2016

29. Glasson SS, Blanchet TJ and Morris EA: The surgical destabilization of the medial meniscus (DMM) model of osteoarthritis in the 129/SvEv mouse. Osteoarthritis Cartilage 15: 1061-1069, 2007.

30. Zhao YG, Wang Y, Guo Z, Gu AD, Dan HC, Baldwin AS, Hao W and Wan YY: Dihydroartemisinin ameliorates inflammatory disease by its reciprocal effects on Th and regulatory $\mathrm{T}$ cell function via modulating the mammalian target of rapamycin pathway. J Immunol 189: 4417-4425, 2012.

31. Zhou S, Wang G, Qiao L, Ge Q, Chen D, Xu Z, Shi D, Dai J, Qin J, Teng H and Jiang Q: Age-dependent variations of cancellous bone in response to ovariectomy in C57BL/6J mice. Exp Ther Med 15: 3623-3632, 2018.

32. Mapp PI and Walsh DA: Mechanisms and targets of angiogenesis and nerve growth in osteoarthritis. Nat Rev Rheumatol 8: 390-398, 2012.

33. Xie H, Cui Z, Wang L, Xia Z, Hu Y, Xian L, Li C, Xie L, Crane J, Wan M, et al: PDGF-BB secreted by preosteoclasts induces angiogenesis during coupling with osteogenesis. Nat Med 20 $1270-1278,2014$

34. Matsuo K and Irie N: Osteoclast-osteoblast communication Arch Biochem Biophys 473: 201-209, 2008.

35. Sims NA and Martin TJ: Coupling the activities of bone formation and resorption: A multitude of signals within the basic multicellular unit. Bonekey Rep 3: 481, 2014.

36. Kamekura S, Hoshi K, Shimoaka T, Chung U, Chikuda H, Yamada T, Uchida M, Ogata N, Seichi A, Nakamura K and Kawaguchi H: Osteoarthritis development in novel experimental mouse models induced by knee joint instability. Osteoarthritis Cartilage 13: 632-641, 2005.

37. Honma M, Ikebuchi Y, Kariya Y and Suzuki H: Regulatory mechanisms of RANKL presentation to osteoclast precursors. Curr Osteoporos Rep 12: 115-120, 2014.

38. Miyatake K, Muneta T, Ojima M, Yamada J, Matsukura Y, Abula K, Sekiya I and Tsuji K: Coordinate and synergistic effects of extensive treadmill exercise and ovariectomy on articular cartilage degeneration. BMC Musculoskelet Disord 17: 238 , 2016.

39. Sniekers YH, Weinans H, Bierma-Zeinstra SM, van Leeuwen JP and van Osch GJ: Animal models for osteoarthritis: The effect of ovariectomy and estrogen treatment-a systematic approach. Osteoarthritis Cartilage 16: 533-541, 2008.

40. Bijlsma JW, Berenbaum F and Lafeber FP: Osteoarthritis: An update with relevance for clinical practice. Lancet 377: 2115-2126, 2011

41. Goldring MB and Goldring SR: Articular cartilage and subchondral bone in the pathogenesis of osteoarthritis. Ann N Y Acad Sci 1192: 230-237, 2010.

42. Bultink IE and Lems WF: Osteoarthritis and osteoporosis: What is the overlap? Curr Rheumatol Rep 15: 328, 2013.

43. Baron R and Kneissel M: WNT signaling in bone homeostasis and disease: From human mutations to treatments. Nat Med 19: 179-192, 2013.
44. Song L, Liu M, Ono N, Bringhurst FR, Kronenberg HM and Guo J: Loss of wnt $/ \beta$-catenin signaling causes cell fate shift of preosteoblasts from osteoblasts to adipocytes. J Bone Miner Res 27: 2344-2358, 2012.

45. van Bezooijen RL, Roelen BA, Visser A, van der Wee-Pals L, de Wilt E, Karperien M, Hamersma H, Papapoulos SE, ten Dijke P and Löwik CW: Sclerostin is an osteocyte-expressed negative regulator of bone formation, but not a classical BMP antagonist. J Exp Med 199: 805-814, 2004.

46. Li X, Ominsky MS, Warmington KS, Morony S, Gong J, Cao J, Gao Y, Shalhoub V, Tipton B, Haldankar R, et al: Sclerostin antibody treatment increases bone formation, bone mass, and bone strength in a rat model of postmenopausal osteoporosis. J Bone Miner Res 24: 578-588, 2009

47. Wu L, Guo H, Sun K, Zhao X, Ma T and Jin Q: Sclerostin expression in the subchondral bone of patients with knee osteoarthritis. Int J Mol Med 38: 1395-1402, 2016.

48. Walker EC, McGregor NE, Poulton IJ, Solano M, Pompolo S, Fernandes TJ, Constable MJ, Nicholson GC, Zhang JG, Nicola NA, et al: Oncostatin M promotes bone formation independently of resorption when signaling through leukemia inhibitory factor receptor in mice. J Clin Invest 120: 582-592, 2010.

49. Poulton IJ, McGregor NE, Pompolo S, Walker EC and Sims NA: Contrasting roles of leukemia inhibitory factor in murine bone development and remodeling involve region-specific changes in vascularization. J Bone Miner Res 27: 586-595, 2012.

50. Johnson RW, Brennan HJ, Vrahnas C, Poulton IJ, McGregor NE, Standal T, Walker EC, Koh TT, Nguyen H, Walsh NC, et al: The primary function of gp130 signaling in osteoblasts is to maintain bone formation and strength, rather than promote osteoclast formation. J Bone Miner Res 29: 1492-1505, 2014.

51. Li X, Ominsky MS, Niu QT, Sun N, Daugherty B, D'Agostin D, Kurahara C, Gao Y, Cao J, Gong J, et al: Targeted deletion of the sclerostin gene in mice results in increased bone formation and bone strength. J Bone Miner Res 23: 860-869, 2008.

52. de Vries TJ and Huesa C: The osteocyte as a novel key player in understanding periodontitis through its expression of RANKL and sclerostin: A review. Curr Osteoporos Rep 17: 116-121, 2019.

53. Dong F, Zhou X, Li C, Yan S, Deng X, Cao Z, Li L, Tang B, Allen TD and Liu J: Dihydroartemisinin targets VEGFR2 via the NF-kB pathway in endothelial cells to inhibit angiogenesis. Cancer Biol Ther 15: 1479-1488, 2014.

54. Ho WE, Peh HY, Chan TK and Wong WS: Artemisinins: Pharmacological actions beyond anti-malarial. Pharmacol Ther 142: 126-139, 2014.

55. Bei MJ, Tian FM, Xiao YP, Cao XH, Liu N, Zheng ZY, Dai MW, Wang WY, Song HP and Zhang L: Raloxifene retards cartilage degradation and improves subchondral bone micro-architecture in ovariectomized rats with patella baja-induced-patellofemoral joint osteoarthritis. Osteoarthritis Cartilage 28: 344-355, 2020.

This work is licensed under a Creative Commons Attribution-NonCommercial-NoDerivatives 4.0 International (CC BY-NC-ND 4.0) License. 